\title{
The Contribution of Crystal Lattice Energy on The Dissolution Behavior of Eutectic Solid Dispersions
}

Kaushalendra Chaturvedi ${ }^{1}$, Harsh S. Shah ${ }^{2}$, Kajal Nahar ${ }^{3}$, Rutesh Dave ${ }^{4}$, Kenneth R. Morris ${ }^{5 *}$

${ }^{1}$ Graduate student at Arnold and Marie Schwartz College of Pharmacy and Health Sciences, Long Island University, Brooklyn, NY-11201; Email: kaushalendra.chaturvedi@my.liu.edu Telephone- (631) 408-2934

${ }^{2}$ Graduate student at Arnold and Marie Schwartz College of Pharmacy and Health Sciences, Long Island University, Brooklyn, NY-11201; Email- harsh.shah3@my.liu.edu;

Telephone- (862) 368-4439

${ }^{3}$ Graduate student at Arnold and Marie Schwartz College of Pharmacy and Health Sciences, Long Island University, Brooklyn, NY-11201; Email- kajalajit.nahar@my.liu.edu Telephone- (929) 354-4616

${ }^{4}$ Professor and Director of the Division of Pharmaceutical Sciences, Arnold and Marie Schwartz College of Pharmacy and Health Sciences, Long Island University, Brooklyn, NY-11201; Email- Rutesh.Dave@,liu.edu; Telephone- (718) 488-1660

5* University Professor and Director of the Lachman Institute for Pharmaceutical Analysis, Arnold and Marie Schwartz College of Pharmacy and Health Sciences, Long Island University, Brooklyn, NY-11201; Email- Kenneth.Morris@liu.edu ; Telephone- (718) 246-6452

*Corresponding author 
Table S1- DSC experimental data from IBU/PEG-6000 system

\begin{tabular}{|c|c|c|c|c|c|c|c|c|c|c|}
\hline \multirow{3}{*}{ Sr.No. } & \multirow{2}{*}{\multicolumn{2}{|c|}{$\%$ of $w / w$}} & \multirow{2}{*}{\multicolumn{2}{|c|}{$\begin{array}{c}\text { Run } 1 \\
\text { Temp at solidus curve } \\
\left({ }^{\circ} \mathrm{C}\right)\end{array}$}} & \multirow{2}{*}{\multicolumn{2}{|c|}{$\begin{array}{c}\text { Run } 2 \\
\text { Temp at solidus curve } \\
\left({ }^{\circ} \mathrm{C}\right)\end{array}$}} & \multirow{2}{*}{\multicolumn{2}{|c|}{$\begin{array}{c}\text { Avg } \\
\text { Temp }\left({ }^{\circ} \mathbf{C}\right)\end{array}$}} & \multirow{2}{*}{\multicolumn{2}{|c|}{$\begin{array}{c} \pm \text { SD } \\
\text { Temp }\left({ }^{\circ} \mathrm{C}\right)\end{array}$}} \\
\hline & & & & & & & & & & \\
\hline & IBU & PEG-6000 & Onset & Peak & Onset & Peak & Onset & Peak & Onset & Peak \\
\hline 1 & 100 & 0 & & & & & & & & \\
\hline 2 & 90 & 10 & 46.900 & 48.620 & 48.780 & 50.920 & 47.840 & 49.770 & 1.329 & 1.626 \\
\hline 3 & 75 & 25 & 48.760 & 51.170 & 46.900 & 48.990 & 47.830 & 50.080 & 1.315 & 1.541 \\
\hline 4 & 50 & 50 & 46.880 & 49.170 & 48.400 & 51.900 & 47.640 & 50.535 & 1.075 & 1.930 \\
\hline 5 & 35 & 65 & 48.910 & 50.950 & 48.610 & 52.160 & 48.760 & 51.555 & 0.212 & 0.856 \\
\hline 6 & 25 & 75 & 46.980 & 49.170 & 49.460 & 52.710 & 48.220 & 50.940 & 1.754 & 2.503 \\
\hline 7 & 0 & 100 & & & & & & & & \\
\hline
\end{tabular}

Table S2- DSC experimental data from APAP/CAFF system

\begin{tabular}{|c|c|c|c|c|c|c|c|c|c|c|}
\hline \multirow[t]{3}{*}{ Sr.No. } & \multirow{2}{*}{\multicolumn{2}{|c|}{$\%$ of $w / w$}} & \multirow{2}{*}{\multicolumn{2}{|c|}{$\begin{array}{c}\text { Run } 1 \\
\text { Temp at solidus curve } \\
\left({ }^{\circ} \mathrm{C}\right)\end{array}$}} & \multirow{2}{*}{\multicolumn{2}{|c|}{$\begin{array}{c}\text { Run } 2 \\
\text { Temp at solidus curve } \\
\left({ }^{\circ} \mathrm{C}\right)\end{array}$}} & \multirow{2}{*}{\multicolumn{2}{|c|}{$\begin{array}{c}\text { Avg } \\
\text { Temp }\left({ }^{\circ} \mathrm{C}\right)\end{array}$}} & \multirow{2}{*}{\multicolumn{2}{|c|}{$\begin{array}{c} \pm \text { SD } \\
\text { Temp }\left({ }^{\circ} \mathrm{C}\right)\end{array}$}} \\
\hline & & & & & & & & & & \\
\hline & APAP & CAFF & Onset & Peak & Onset & Peak & Onset & Peak & Onset & Peak \\
\hline 1 & 100 & 0 & & & & & & & & \\
\hline 2 & 90 & 10 & 133.35 & 139.31 & 132.41 & 140.82 & 132.880 & 140.065 & 0.665 & 1.068 \\
\hline 3 & 80 & 20 & 134.31 & 139.21 & 132.41 & 140.82 & 133.360 & 140.015 & 1.344 & 1.138 \\
\hline 4 & 60 & 40 & 129.37 & 139.6 & 131.46 & 141.39 & 130.415 & 140.495 & 1.478 & 1.266 \\
\hline 5 & 20 & 80 & 134.99 & 139.63 & 132.99 & 141.01 & 133.990 & 140.320 & 1.414 & 0.976 \\
\hline 6 & 10 & 90 & 133.63 & 138.53 & 135.55 & 140.88 & 134.590 & 139.705 & 1.358 & 1.662 \\
\hline 7 & 0 & 100 & & & & & & & & \\
\hline
\end{tabular}

